\title{
HIGHLIGHT
}

\section{Protein kinase A in Carney complex: a new example of cAMP pathway alteration in endocrine tumors}

\author{
Jérôme Bertherat \\ Service d'endocrinologie and CNRS UPR 1524, CHU Cochin, 27 rue du Fg-St-Jacques, 75014, Paris, France \\ (Correspondence should be addressed to J Bertherat; E-mail: jerome.bertherat@cch.ap-hop-paris.fr)
}

Cyclic AMP (cAMP) plays an important role in cellular differentiation and has a complex action on cellular proliferation. Activation of the cAMP pathway inhibits cellular proliferation in numerous tissues, but it can also stimulate cellular proliferation in a subset of endocrine cells. For instance, cAMP increases somatotroph and thyroid cells proliferation (1). In these tissues, cAMP is also the well known intracellular mediator of peptidic hormones (growth hormone releasing hormone (GHRH) and thyroid stimulating hormone (TSH) respectively) which play a major role in development and differentiation. These hormones bind to seven transmembranes receptors coupled to $G$ stimulatory (Gs) protein. Inactivating mutation of several receptors coupled to Gs, such as the GHRH, adrenocorticotropin (ACTH) or TSH receptors, lead to hormone resistance and somatotroph, adrenal or thyroid deficiency respectively. Normal function of the cAMP pathway from the cell surface to the nucleus seems to be required for normal development of both the thyroid and the somatotrophs. Expression of a dominant negative mutant of the cAMP response element binding protein (CREB) non-phosphorylable by protein kinase A (PKA) causes hypoplasia of the targeted organ and thyroid or somatotroph deficiency in transgenic mice (2).

Several diseases with endocrine gland hyperplasia or tumor, and hormone hypersecretion, can be secondary to activation of the extracellular signal that stimulates the cAMP pathway in a given tissue. Eutopic or ectopic GHRH or ACTH hypersecretion cause acromegaly with somatotroph hyperplasia or Cushing's syndrome with adrenal bilateral hyperplasia. Pituitary tumors secreting TSH lead to the development of goiter and hyperthyroidism. Several molecular alterations mimicking overstimulation of the cAMP pathway by extracellular stimuli have been observed in various endocrine tumors. Somatic activating mutations of the TSH receptor causing ligand independent activation of cAMP production by adenylyl cyclase are observed in most thyroid toxic adenomas (3). In these tumors, activation of the cAMP pathway could be linked to both thyroid hormone hypersecretion and cellular proliferation leading to adenoma development. Germ line inheritance of similar activating TSH receptor mutations have been observed in the dominant autosomic form of toxic nodular goiter. In the adrenal cortex, ectopic expression of the gastric inhibitory peptide receptor, also coupled to Gs, is associated with ACTHindependent macronodular adrenal hyperplasia or adenoma and food-dependent cortisol hypersecretion (4). Alterations of the Gs protein itself were reported more than 10 years ago in GH-secreting and thyroid tumors. These somatic activating Gs mutations (oncogene Gsp) lead to constitutive cAMP production (5). Similar mutations have also been reported in the McCune-Albright syndrome, presenting with 'café au lait' spot, bone fibrous dysplasia and various endocrine tumors: growth hormone (GH)-secreting adenomas, precocious puberty with ovarian cyst, toxic thyroid goiter, Cushing's syndrome with ACTH-independent macronodular adrenal hyperplasia. Patients suffering from the McCune-Albright syndrome present with a mosaic distribution of the Gsp mutation (6).

Carney complex (CC) is an autosomic dominant disorder initially described as the association of myxomas, spotty skin pigmentation and endocrine overactivity and considered as a multiple neoplasia and lentiginosis syndrome. Various endocrine tumors are associated with the CC: GH-secreting adenomas, thyroid toxic adenomas, Sertoli cell testicular tumors, primary pigmented nodular adrenal hyperplasia with ACTH-independent Cushing's syndrome. Cardiac myxomas and psammomatous melanotic schwannomas are among other various tumors observed in the CC (7). The CC share some features of the McCune-Albright syndrome. Because of these similarities genes controlling the cAMP pathway have been considered as candidates for CC. Mutation of Gs protein has not been found in CC. Two loci have been identified by linkage analysis in CC families. By this approach the putative genes responsible for $\mathrm{CC}$ have been mapped to chromosome 2 p16 and 17q22-24. Genomic amplification of the $2 \mathrm{p} 16$ region in tumors from $\mathrm{CC}$ patients suggest that an oncogene is located at this locus. On the other hand loss of heterozygosity $(\mathrm{LOH})$ of the $17 q 22-24$ region in tumors from CC patients suggest that a tumor suppressor gene is located at this other locus. Study of $\mathrm{LOH}$ in CC tumors allowed C Stratakis and his team to redefine a $8-\mathrm{cM}$ region at the $17 \mathrm{q} 22$ 24 locus that retains the allele segregating with the disease. Among the known genes located in this region is the one encoding the regulatory subunit $1 \mathrm{~A}$ of the cAMP dependent protein kinase (PKAR1A). Two 


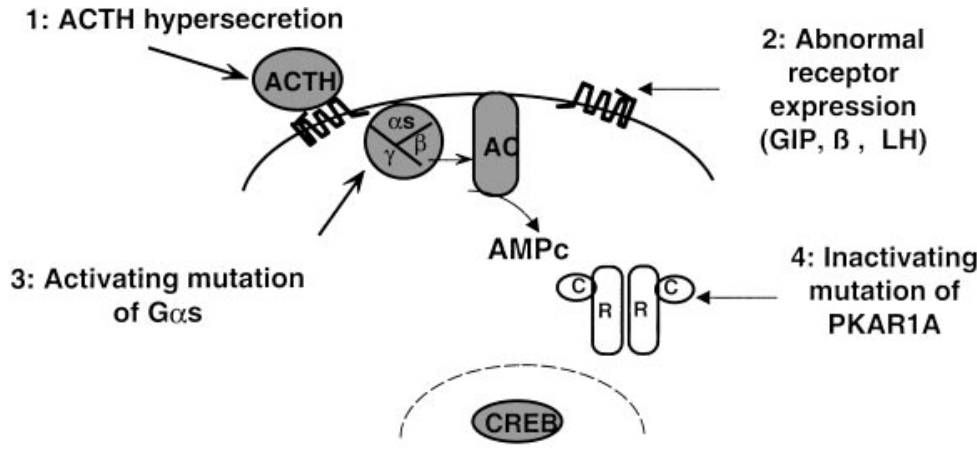

Figure 1 Mechanisms of cAMP pathway activation in Cushing's syndrome. The figure shows alterations of the CAMP pathway at the extracellular (1), as well as the intracellular $(2,3,4)$ level in various forms of Cushing's syndrome associated with adrenal hyperplasia, adrenal tumors, macro- or micro-nodular adrenocortical hyperplasia. 1. Eutopic (Cushing's disease) or ectopic ACTH secretion. 2. Abnormal expression of membrane receptor: GIP (gastric-inhibitory peptide) receptor, $\beta$-adrenergic receptor $(\beta)$, luteinizing hormone receptor (LH). 3. Activating mutation of the $\alpha$ subunit of the Gs protein, termed oncogene Gsp in McCune-Albright syndrome. 4. Inactivating mutation of the PKAR1A in Carney complex. AC, adenylyl cyclase; $\mathrm{C}$, catalytic, $\mathrm{R}$; regulatory subunit.

groups recently reported evidence that the PKAR1A is the CC susceptibility gene located on 17q22-24. Kirschner and collaborators found an heterozygous PKAR1A gene mutation in six unrelated kindred and one sporadic case with typical features of CC (8). Casey and collaborators also reported a heterozygous mutation of the same gene in three unrelated kindred, who mainly presented with spotty pigmentation of the skin and cardiac myxomas (9). The sporadic case reported by L S Kirschner presented with the same mutation as three kindred with CC, while the other families all had different mutations. The mutations are most often a one or two base pair deletion located in exons 4 to 8 of the PKAR1A gene that contains 11 exons. These mutations were not observed in unaffected individuals in CC families nor in 100 normal unrelated subjects. Most of these alterations of the PKAR1A gene cause frameshift leading to premature stop codon and are likely to be inactivating mutations generating a truncated protein that does not contain the two cAMP binding sites observed in the wild type R1A.

PKA is a tetramer consisting of two regulatory and two catalytic subunits. Four genes encoding the regulatory subunit have been identified: R1A, R1B, $\mathrm{R} 2 \mathrm{~A}, \mathrm{R} 2 \mathrm{~B}$. The holoenzyme is the inactive tetramer. After cAMP binding to the regulatory subunit, the catalytic subunits dissociate and become active. The free catalytic subunit therefore phosphorylates serine and threonine residues and is also able to enter the cell nucleus to regulate gene expression by transcription factor phosphorylation. The equilibrium between the various components of the PKA holoenzyme is complex and might be different among the tissues, but could also change during development or the cell cycle. PKAR1A normally inhibits PKA activity, therefore inactivation of this regulatory subunit by the CC mutation is expected to increase PKA activity if no compensatory mechanisms occur. Interestingly, study of the PKA regulatory subunits R1A and R2B in CC tumors by Western blot analysis showed a reduced R1A level without compensatory R2B overexpression (9). Therefore, the ratio of $\mathrm{R} 1 \mathrm{~A} / \mathrm{R} 2 \mathrm{~B}$ is altered and these observations suggest that $\mathrm{CC}$ mutations lead to haploinsufficiency of PKAR1A. Study of PKA activity in CC tumors showed no change in basal activity compared with sporadic tumors from patients not affected by $\mathrm{CC}$, but an increased PKA activity in response to cAMP was observed in CC tumors (8). An increased PKA activity in endocrine tumors of the $\mathrm{CC}$ is consistent with other molecular defects leading to cAMP pathway activation in sporadic GH-secreting or thyroid adenomas (both are endocrine tissues in which cAMP stimulates cell proliferation). Whether inactivating mutations of PKAR1A might have opposite effects in tissues where cAMP inhibits cellular proliferation is an interesting hypothesis that might be supported by a fine tissue specific regulation of the various regulatory subunits gene expression. Since PKA also stimulates tyrosine hydroxylase gene expression through phosphorylation of the transcription factor CREB, it might be interesting to speculate that increased skin pigmentation observed in CC might be explained by increased cutaneous PKA activity leading to stimulation of melanin biosynthesis.

So far the various molecular defects affecting the cAMP pathway reported in endocrine tumors altered gene encoding proteins located at the cell membrane level (i.e. receptors and G protein, Fig. 1). The report of PKAR1A mutations is the first example of a downstream defect in this pathway. Since the identification of the second CC susceptibility gene located on the $2 \mathrm{p} 16$ gene in other families has not yet been reported, it is tempting to speculate that this other $\mathrm{CC}$ gene could also control the cAMP pathway.

\section{References}

1 Bertherat J. Nuclear effects of the cAMP pathway activation in somatotrophs. Hormone Research 199747 245-250.

2 Struthers RS, Vale WW, Arias C, Sawchenko PE \& Montminy MR. Somatotroph hypoplasia and dwarfism in transgenic mice expressing a non-phosphorylable CREB mutant. Nature 1991 $350622-624$.

3 Parma J, Duprez L, Van Sande J, Cochaux P, Gervy C, Mockel J, Dumont J \& Vassart G. Somatic mutations in the thyrotropin 
receptor gene cause hyperfunctioning thyroid adenomas. Nature $1993365649-651$.

4 Lacroix A, Bolte E, Tremblay J, Dupre J, Poitras P, Fournier H et al. Gastric inhibitory polypeptide-dependent cortisol secretion in macronodular adrenal hyperplasia: a new etiology of Cushing's syndrome. New England Journal of Medicine 1992327 974-980.

5 Landis CA, Masters SB, Spada A, Pace AM, Bourne HR \& Vallar L. GTPase inhibiting mutations activate the alpha chain of Gs and stimulates adenylyl cyclase in human pituitary tumors. Nature $1989340692-696$.

6 Weinstein LS, Shenker A, Gejman PV, Merino MJ, Friedman E \& Spiegel AM. Activating mutations of the stimulatory G protein in the McCune-Albright syndrome. New England Journal of Medicine $19913251688-1695$.

7 Stratakis CA, Carney JA, Lin JP, Papanicolaou DA, Karl M, Kastner DL et al. Carney complex, a familial multiple neoplasia and lentoginosis syndrome: analysis of 11 kindreds and linkage to the short arm of chromosome 2. Journal of Clinical Investigation 199697 699-705.

8 Kirschner LS, Carney JA, Pack SD, Taymans SE, Giatzakis C, Cho YS et al. Mutations of the gene encoding the protein kinase A type 1-a regulatory subunit in patients with the Carney complex. Nature Genetics 200026 89-92.

9 Casey M, Vaughan CJ, He J, Hatcher CJ, Winter JM, Weremowicz S, et al. Mutations in the protein kinase A R1a regulatory subunit cause familial cardiac myxomas and Carney complex. Journal of Clinical Investigation 2000106 R31-R38.

Received 24 November 2000

Accepted 27 November 2000 